This item was submitted to Loughborough's Research Repository by the author.

Items in Figshare are protected by copyright, with all rights reserved, unless otherwise indicated.

\title{
Parametric identification of vehicle handling using an extended Kalman filter
}

PLEASE CITE THE PUBLISHED VERSION

http://dx.doi.org/10.1504/IJVAS.2007.016404

PUBLISHER

(c) Inderscience Publishers

VERSION

AM (Accepted Manuscript)

LICENCE

CC BY-NC-ND 4.0

REPOSITORY RECORD

Best, Matt C.. 2011. "Parametric Identification of Vehicle Handling Using an Extended Kalman Filter". figshare. https://hdl.handle.net/2134/8317. 
This item was submitted to Loughborough's Institutional Repository (https://dspace.lboro.ac.uk/) by the author and is made available under the following Creative Commons Licence conditions.

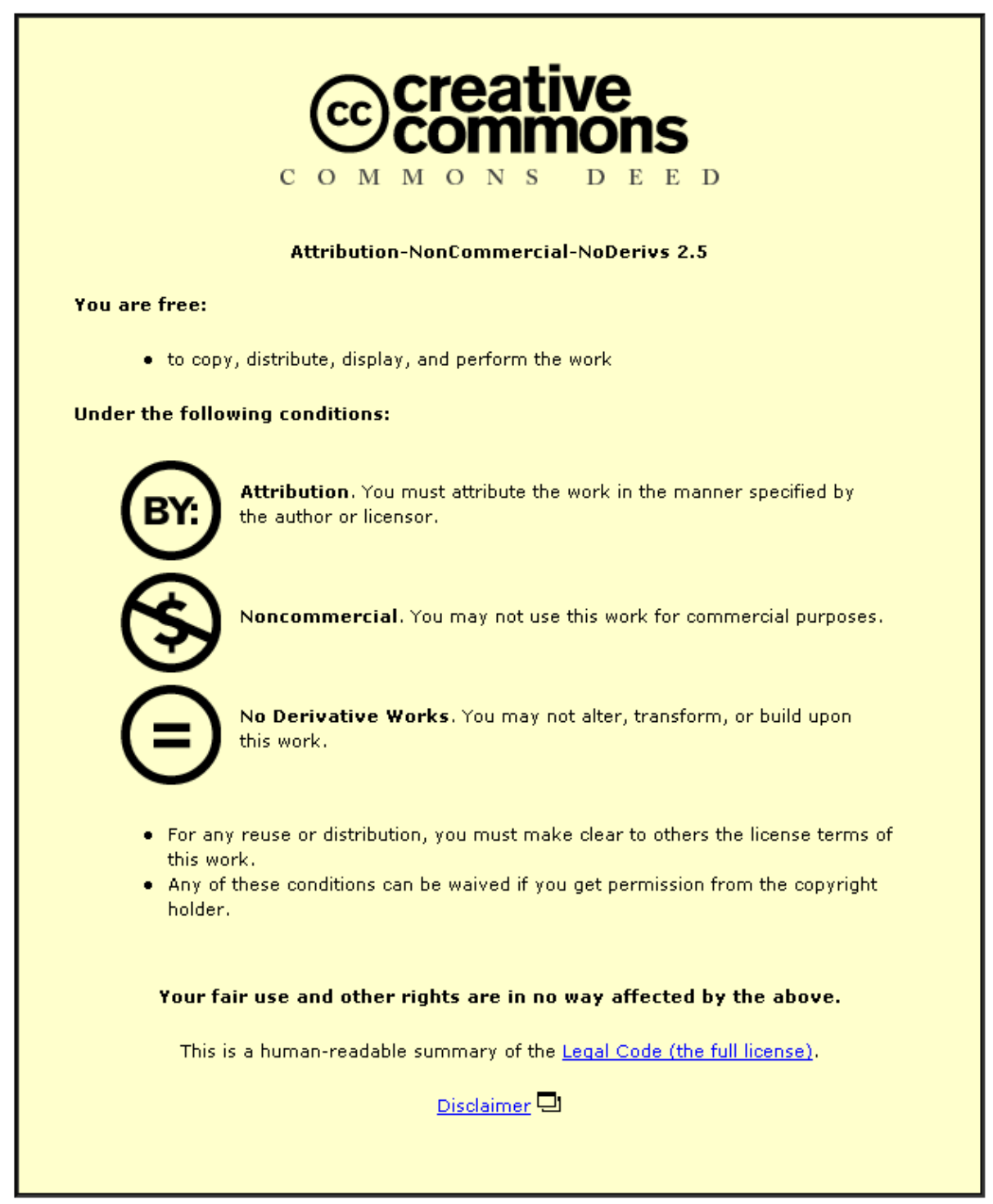

For the full text of this licence, please go to: http://creativecommons.org/licenses/by-nc-nd/2.5/ 


\title{
Parametric Identification of Vehicle Handling using an Extended Kalman Filter
}

\author{
Matthew C Best \\ Dept. Aeronautical and Automotive Engineering \\ Loughborough University \\ Leicestershire, UK
}

LE11 3TU

Email : M.C.Best@lboro.ac.uk

\begin{abstract}
This paper considers a novel method for estimating parameters in a vehicle handling dynamic model using a recursive filter. The well known extended Kalman filter which is widely used for real-time state estimation of vehicle dynamics - is used here in an unorthodox fashion; a model is prescribed for the sensors alone, and the state vector is replaced by a set of unknown model parameters. With the aid of two simple tuning parameters, the system self-regulates its estimates of parameter and sensor errors, and hence smoothly identifies optimal parameter choices. In a linear-in-the-parameters example, the results are shown to be comparable to least-squares identification, but the system works equally well for the more general nonlinear handling model examples, and should be well suited to any smoothly nonlinear system. Moreover, it is shown that by simple adjustment of the tuning parameters the filter can operate in a real-time capacity.
\end{abstract}

Keywords: Vehicle Handling Dynamics, Kalman Filter, System Identification, Vehicle Parameter

Biographical notes: Matt Best is a senior lecturer in vehicle dynamics and control in the department of Aeronautical and Automotive Engineering at Loughborough University in the UK. He gained his $\mathrm{PhD}$ in practical implementation of vehicle ride control at Loughborough in 1995, and has been a lecturer since 1996. His principal research interests include Kalman filter methods for estimation and identification, design of nonlinear optimal observers and controllers for vehicle ride and handling, and vehicle dynamics modelling and simulation. $\mathrm{He}$ has published approx. 30 papers in his field, in conferences, and journals such as Proc. ImechE parts D and K, International Journal of Mechanics and Mobility, and International Journal of Vehicle Design.

\section{NOTATION}

\section{Dynamic Variables}

$r \quad$ yaw rate $(\mathrm{rad} / \mathrm{s})$

$v \quad$ sideslip velocity $(\mathrm{m} / \mathrm{s})$

$u \quad$ forward velocity $(\mathrm{m} / \mathrm{s})$

$\delta \quad$ (front) wheel steer angle (rad)

y sensors vector

$\mathbf{x}$ state vector

$\boldsymbol{\theta}$ parameters vector

$\wedge \quad$ caret denotes estimated signal

$\mathrm{k}, \mathrm{f}, \mathrm{r} \quad$ subscripts denote time step, front and rear respectively $\underline{\text { Kalman Filter }}$

$\omega \quad$ process (model) error

$v \quad$ sensors error

K Optimal gain matrix

$\mathbf{P} \quad$ state error covariance matrix

R sensors error covariance matrix

$\mathbf{Q} \quad$ process error covariance matrix

S sensors / process error cross covariance matrix

$\mathbf{f}(\mathbf{x}) \quad$ nonlinear system model

$\mathbf{h}(\mathbf{x}) \quad$ nonlinear sensors model 


$\begin{array}{llll}\mathbf{F}(\mathbf{x}) & \text { system model Jacobian } & \lambda & \text { parameter error scaling factor } \\ \mathbf{H}(\mathbf{x}) & \text { sensors model Jacobian } & \tau & \text { moving average time constant for noise } \\ T & \text { filter sampling interval (s) } & & \text { matrix 'memory' }\end{array}$

Identifying Kalman Filter

\section{INTRODUCTION}

The Kalman filter has emerged as a popular means of estimating the vector of variables required for state feedback control in the often separated fields of vehicle handling control (eg Arndt et al (2005), Wenzel et al (2006)) and vehicle ride control (eg Best and Gordon (1994)). However, in the emerging field of integrated chassis control, the Kalman filter is even more relevant and powerful. In addition to estimating the states, the filter also embodies a simplified model for the rigid body dynamics to be controlled. This model mirrors the behaviour of the vehicle, and provides a reference for control actions - eg in Hancock et al (2005) and more generally in model reference adaptive controllers, eg $\mathrm{Qu}$ and $\mathrm{Zu}$ (2005). Perhaps most importantly for achieving integrated vehicle control, this single simplified model can describe the whole vehicle, and hence inform the combination of powertrain, handling and ride controls. It can provide a tool to understand potential control combination benefits and conflicts, possibly in real-time, and certainly within the design environment.

The accuracy of the model will directly influence the accuracy of state reconstruction within the Kalman filter, and also the value of the model within controller design and/or operation. Now both on and off the vehicle, the available computing power constrains the complexity of this model, and so ever more complex models are becoming feasible; however there is a strong motivation for maintaining a low order to this model. More complex models require more parameters, and accuracy depends directly on their appropriate choice. Also, conditions such as vehicle weight, tyre wear and pressure, and road friction are time varying, so must be estimated as part of the observer / controller combination. The situation can readily arise where parameter estimation is compromised due to the large number of parameters to be adapted, and their relatively poor rank independence within the estimation algorithm. The astute choice is to keep the model complexity as low as possible, while continually adapting a subset of parameters to match the prevailing conditions. This system identification process is then key to the timely provision of a good model, and it can be used to good effect for all aspects within the integrated controller. In this paper we carry out system identification using an extended Kalman filter structure, as this allows the required nonlinearity in the vehicle model.

The method is developed using a bold, and perhaps contentious assumption however - that the state vector is fully available within the sensor set. Critically, this includes knowledge of the traditionally hard to measure lateral velocity state. However, recent developments of combined differential GPS / inertial measurement systems make this assumption more reasonable, with commercially available (albeit expensive) systems currently declaring accuracies of $0.1 \mathrm{~m} / \mathrm{s}$ in lateral velocity.

The Identifying Extended Kalman Filter (IEKF) operates in an unconventional way, in that the model is restricted to the prediction of the sensor set. The innovation sequence (model vs measured sensor errors) is then used to modify a 'state' vector which is defined as the subset of parameters to be identified. The basic premise is that the available sensors are assumed to include the true state of the vehicle, and the best model parameters are then derived from this 
- a standard assumption in the process of system identification, but novel in its execution by Kalman filter. The method works well, particularly in that it addresses three factors which other, more common system identifications processes lack :

i) Any subset of parameters can be identified, within a general nonlinear form of model. The only restrictions are that the model must be smoothly nonlinear, and the parameters must be suitably independent of each other.

ii) The filter self-regulates and depends on just two tuning parameters, one of which can be set nominally, using simple assumptions about the test inputs.

iii) By tuning the one remaining parameter, the filter can operate with a long or short time constant. Thus it can iteratively extract the best average model to fit a data set, or it can operate in real-time to rapidly adapt the parameters.

The IEKF design is first outlined in Section 2. It is then examined in the simulation environment, with a two degree of freedom nonlinear handling model (Section 3) identified from data generated on a more complex model in Section 4; the models and IEKF design method are introduced, and a sequence of experiments is then conducted to set out the operation and scope of the method. Within this, the operation is examined with two separate parameter sets, and in both slow and fast adaptation modes. The new system is also compared with Ordinary Least Squares (OLS) identification in a linear example, and its use in combination with a filter for real-time state estimation is explored. Perhaps most critically, given the sensor requirements, the filter's resistance to measurement errors is also examined.

\section{IDENTIFYING EXTENDED KALMAN FILTER (IEKF)}

The standard extended Kalman filter (EKF) operates on nonlinear system and sensor models $\mathbf{f}$ and $\mathbf{h}$, which relate the true state vector $\overline{\mathbf{x}}$, measured sensor set $\mathbf{y}$, known inputs $\mathbf{u}$ and model parameters $\boldsymbol{\theta}$ at any instant $\mathrm{k}$ according to

$$
\begin{aligned}
\dot{\overline{\mathbf{x}}}_{k} & =\mathbf{f}\left(\overline{\mathbf{x}}_{k}, \mathbf{u}_{k}, \boldsymbol{\theta}_{k}\right)+\boldsymbol{\omega}_{k} \\
\mathbf{y}_{k} & =\mathbf{h}\left(\overline{\mathbf{x}}_{k}, \mathbf{u}_{k}, \boldsymbol{\theta}_{k}\right)+\mathbf{v}_{k}
\end{aligned}
$$

(see for example Gelb (1974)). $\boldsymbol{\omega}$ thus describes the state propagation modelling error, and $v$ gives the sensor error. $\boldsymbol{v}$ is often misleadingly referred to as the measurement error, when in reality it aggregates measurement noise within $\mathbf{y}$, and sensor modelling errors in $\mathbf{h}$.

An optimal filter can be derived if the error sequences obey the following

$$
\begin{aligned}
& E\left(\boldsymbol{\omega}_{k}\right)=\mathbf{0}, \quad E\left(\boldsymbol{v}_{k}\right)=\mathbf{0}, \quad \forall k \\
& E\left(\boldsymbol{\omega}_{i} \boldsymbol{\omega}_{j}^{T}\right)=\mathbf{0}, \quad E\left(\mathbf{v}_{i} \mathbf{v}_{j}^{T}\right)=\mathbf{0}, \quad \forall(i \neq j) \\
& \mathbf{Q}_{k}=E\left(\boldsymbol{\omega}_{k} \boldsymbol{\omega}_{k}^{T}\right), \quad \mathbf{S}_{k}=E\left(\boldsymbol{\omega}_{k} \boldsymbol{v}_{k}^{T}\right)=\mathbf{0}, \quad \mathbf{R}_{k}=E\left(\boldsymbol{v}_{k} \mathbf{v}_{k}^{T}\right)
\end{aligned}
$$

where the error covariance matrices $\mathbf{Q}_{k}, \mathbf{R}_{k}$ and $\mathbf{S}_{k}$ are assumed known. In practice they are difficult to estimate and their setting is a topic of continuing interest (see for example, Haykin (2001)). They are often assumed to be time-invariant, and are approximated, or even set nominally, with $\mathbf{S}$ often neglected as approximately zero. In this application we will see only 
initial conditions required for these matrices, with the true covariances being identified within the algorithm.

The EKF also requires model Jacobians to be evaluated at each time step, defined

$$
\begin{aligned}
\mathbf{F}\left(\hat{\mathbf{x}}_{k}\right) & =\left.\frac{\partial \mathbf{f}\left(\mathbf{x}, \mathbf{u}_{k}, \boldsymbol{\theta}_{k}\right)}{\partial \mathbf{x}}\right|_{\mathbf{x}=\hat{\mathbf{x}}_{k}} \\
\mathbf{H}\left(\hat{\mathbf{x}}_{k}\right) & =\left.\frac{\partial \mathbf{h}\left(\mathbf{x}, \mathbf{u}_{k}, \boldsymbol{\theta}_{k}\right)}{\partial \mathbf{x}}\right|_{\mathbf{x}=\hat{\mathbf{x}}_{k}}
\end{aligned}
$$

and the full set of equations for the standard, real-time state estimation application are

$$
\begin{gathered}
\mathbf{F}_{k}^{*}=\mathbf{F}\left(\hat{\mathbf{x}}_{k}\right)-\mathbf{S} \mathbf{R}^{-1} \mathbf{H}\left(\hat{\mathbf{x}}_{k}\right) \\
\mathbf{K}_{k}=\mathbf{P}_{k} \mathbf{H}^{\mathbf{T}}\left(\hat{\mathbf{x}}_{k}\right)\left[\mathbf{H}\left(\hat{\mathbf{x}}_{k}\right) \mathbf{P}_{k} \mathbf{H}^{\mathbf{T}}\left(\hat{\mathbf{x}}_{k}\right)+\mathbf{R}\right]^{-1} \\
\mathbf{P}_{k}^{*}=\left[\mathbf{I}-\mathbf{K}_{k} \mathbf{H}\left(\hat{\mathbf{x}}_{k}\right)\right] \mathbf{P}_{k} \\
\mathbf{P}_{k+1}=\mathbf{P}_{k}^{*}+T\left[\mathbf{Q}-\mathbf{S} \mathbf{R}^{-1} \mathbf{S}^{\mathbf{T}}+\mathbf{F}^{*}\left(\hat{\mathbf{x}}_{k}\right) \mathbf{P}_{k}^{*}+\mathbf{P}_{k}^{*} \mathbf{F}^{* \mathbf{T}}\left(\hat{\mathbf{x}}_{k}\right)\right] \\
\hat{\mathbf{x}}_{k+1}=\hat{\mathbf{x}}_{k}+\mathbf{K}_{k}\left(\mathbf{y}_{k}-\mathbf{h}\left(\hat{\mathbf{x}}_{k}\right)\right)+T\left[\mathbf{f}\left(\hat{\mathbf{x}}_{k}\right)+\mathbf{S} \mathbf{R}^{-1}\left(\mathbf{y}_{k}-\mathbf{h}\left(\hat{\mathbf{x}}_{k}\right)\right)\right]
\end{gathered}
$$

where the filter sample time $T$ is used to provide a simple Euler integration of the state derivatives.

Now the premise adopted in Best et al (2000) is that an EKF can have its state vector augmented to include a subset of the model parameters. The resulting filter assumes no known model for the parameter variation, and simply ensures slow adaptation by adjusting the expectation of errors related to the parameter changes; so Equation (1) becomes

$$
\dot{\overline{\mathbf{z}}}_{k}=\left[\begin{array}{c}
\dot{\overline{\mathbf{x}}}_{k} \\
\dot{\overline{\boldsymbol{\theta}}}
\end{array}\right]=\left[\begin{array}{c}
\mathbf{f}\left(\mathbf{x}_{k}, \mathbf{u}_{k}, \boldsymbol{\theta}_{k}\right) \\
\mathbf{0}
\end{array}\right]+\left[\begin{array}{c}
\boldsymbol{\omega}_{k}^{(\mathbf{x})} \\
\boldsymbol{\omega}_{k}^{(\boldsymbol{\theta})}
\end{array}\right]
$$

and the covariance $E\left(\boldsymbol{\omega}_{k}^{(\boldsymbol{\theta})} \boldsymbol{\omega}_{k}^{(\boldsymbol{\theta}) T}\right)$ is then set as a tuning parameter, to adjust the rate of adaptation, ensuring this is 'slow' compared to the state propagation dynamics. This method of combining state and parameter identification is attractive, and Best et al (2000) shows that it can be effective - there is just some concern about limitations due to the combination of fast and slow dynamic responses, and the setting of error covariances.

The proposed identifying Kalman filter IEKF takes the formulation one step further; provided the state vector is entirely represented as a subset of the sensor set, $\mathbf{x}_{k} \in \mathbf{y}_{k}$ (which was formerly difficult to achieve in the vehicle handling dynamics context, but which may now be possible given the new generation GPS / inertial measurement systems) we can form the state vector entirely as a set of the parameters, such that Equations (1) and (2) become

$$
\begin{gathered}
\dot{\boldsymbol{\theta}}_{k}=\boldsymbol{\omega}_{k} \\
\mathbf{y}_{k}=\mathbf{h}\left(\mathbf{y}_{k-1}, \mathbf{u}_{k-1}, \boldsymbol{\theta}_{k-1}\right)+\mathbf{v}_{k}
\end{gathered}
$$


where here the sensor equation is simply modified to include an Euler integrated propagation of each variable over a time step, to avoid identity equations. This reduces the system such that the entire model is represented within $\mathbf{h}$ alone. Note however that it also reduces the system to a form where the error covariance matrices can be determined from the noise sequences $\boldsymbol{\omega}_{k}$ and $\boldsymbol{v}_{k}$, which are now directly calculable. The form of Equations (1) and (2) depends on the unknown $\overline{\mathbf{x}}_{k}$, so the error covariances cannot be explicitly determined within that filter - hence in other Kalman filter applications, $\mathbf{Q}, \mathbf{R}$ and $\mathbf{S}$ are design matrices, the choice of which strongly influences the success of the resulting filter.

The IEKF propagates its own error covariances, so $\mathbf{Q}_{k}, \mathbf{R}_{k}$ and $\mathbf{S}_{k}$ are now time varying. Applying Equations (13) and (14) to the EKF formulae of Equations (7) - (11), and noting that now $\mathbf{f}=\mathbf{0}$ and $\mathbf{F}=\mathbf{0}$, we have

$$
\begin{gathered}
\mathbf{H}\left(\hat{\boldsymbol{\theta}}_{k}\right)=\left.\frac{\partial \mathbf{h}\left(\mathbf{x}_{k}, \mathbf{u}_{k}, \boldsymbol{\theta}\right)}{\partial \boldsymbol{\theta}}\right|_{\boldsymbol{\theta}=\hat{\boldsymbol{\theta}}_{k}} \\
\mathbf{K}_{k}=\mathbf{P}_{k} \mathbf{H}^{\mathbf{T}}\left(\hat{\boldsymbol{\theta}}_{k}\right)\left[\mathbf{H}\left(\hat{\boldsymbol{\theta}}_{k}\right) \mathbf{P}_{k} \mathbf{H}^{\mathbf{T}}\left(\hat{\boldsymbol{\theta}}_{k}\right)+\mathbf{R}_{k}\right]^{-1} \\
\mathbf{P}_{k}^{*}=\left[\mathbf{I}-\mathbf{K}_{k} \mathbf{H}\left(\hat{\boldsymbol{\theta}}_{k}\right)\right] \mathbf{P}_{k} \\
\mathbf{P}_{k+1}=\mathbf{P}_{k}^{*}+T\left[\mathbf{Q}_{k}-\mathbf{S}_{k} \mathbf{R}_{k}^{-1} \mathbf{S}_{k}^{T}-\mathbf{S}_{k} \mathbf{R}_{k}^{-1} \mathbf{H}\left(\hat{\boldsymbol{\theta}}_{k}\right) \mathbf{P}_{k}^{*}+\mathbf{P}_{k}^{*} \mathbf{H}\left(\hat{\boldsymbol{\theta}}_{k}\right) \mathbf{R}_{k}^{-1} \mathbf{S}_{k}^{T}\right] \\
\hat{\boldsymbol{\theta}}_{k+1}=\hat{\boldsymbol{\theta}}_{k}+\left(\mathbf{K}_{k}+T \mathbf{S}_{k} \mathbf{R}_{k}^{-1}\right)\left(\mathbf{y}_{k}-\mathbf{h}\left(\mathbf{y}_{k}, \mathbf{u}_{k}, \hat{\boldsymbol{\theta}}_{k}\right)\right)
\end{gathered}
$$

where,

$$
\begin{gathered}
\mathbf{Q}_{k+1}=(1-\alpha) \mathbf{Q}_{k}+\alpha \lambda^{2} \boldsymbol{\omega}_{k} \boldsymbol{\omega}_{k}^{T} \\
\mathbf{S}_{k+1}=(1-\alpha) \mathbf{S}_{k}+\alpha \lambda \boldsymbol{\omega}_{k} \mathbf{v}_{k}^{T} \\
\mathbf{R}_{k+1}=(1-\alpha) \mathbf{R}_{k}+\alpha \mathbf{v}_{k} \mathbf{v}_{k}^{T}
\end{gathered}
$$

with

$$
\begin{gathered}
\boldsymbol{\omega}_{k}=\frac{1}{T}\left(\hat{\boldsymbol{\theta}}_{k+1}-\hat{\boldsymbol{\theta}}_{k}\right) \\
\mathbf{v}_{k}=\mathbf{y}_{k+1}-\mathbf{h}\left(\mathbf{y}_{k}, \mathbf{u}_{k}, \hat{\boldsymbol{\theta}}_{k}\right)
\end{gathered}
$$

Apart from suitable nominal initial conditions for $\mathbf{Q}_{0}, \mathbf{R}_{0}$ and $\mathbf{S}_{0}$, two tuning parameters are now required, $\alpha$ and $\lambda$. $\alpha$ applies an exponentially weighted moving average to the propagation of the noise matrices in order to introduce an appropriate memory of the error history into the covariance. It can better be interpreted in terms of the filtering time constant, $\tau$ it introduces, using

$$
\alpha=1-e^{-\frac{T}{\tau}}
$$

$\lambda$ performs a similar function to the design covariance $E\left(\boldsymbol{\omega}_{k}^{(\boldsymbol{\theta})} \boldsymbol{\omega}_{k}^{(\boldsymbol{\theta}) T}\right)$, in Best et al (2000). Set in the range $0<\lambda<1$, it diminishes the expectation of error in the change in parameters, stabilising the identification. Put simply, the filter causes parameter adaptation which induces 
(a desirable) non-zero $\dot{\boldsymbol{\theta}}_{k}$. However, these changes are errors according to the zero model of Equation (13), and if their total magnitude is interpreted as error, $\mathbf{Q}_{\mathrm{k}}$ becomes relatively large compared with $\mathbf{R}_{\mathrm{k}}$, which results in an increase in the feedback gain $\mathbf{K}_{\mathrm{k}}$ to provide greater correction to the $\theta$. Subsequent parameter corrections are then larger, and this induces instability. $\lambda$ provides a means of balancing the filter such that changes in $\mathbf{Q}_{\mathrm{k}}$ are, correctly, not interpreted entirely as error.

\section{SIMULATION MODELS}

Here we seek a means of testing the method within a known environment to explore variations of tuning parameters and define measures of success. A nonlinear identification model is clearly required, so the simple 'bicycle' model, with Pacejka tyre nonlinearity is used to illustrate the method:

$$
\begin{gathered}
\dot{v}=\left(F_{y f}+F_{y r}\right) / m M_{0}-u r \\
\dot{r}=\left(a F_{y f}-(L-a) F_{y r}\right) / i I_{Z Z 0}
\end{gathered}
$$

with front and rear tyre forces aggregated over the two tyres at each axle,

$$
F_{y i}=p_{i} F_{0} \sin \left[c_{i} \tan ^{-1}\left\{\bar{\alpha}_{i}-e_{i}\left(\bar{\alpha}_{i}-\tan ^{-1}\left(\bar{\alpha}_{i}\right)\right)\right\}\right]
$$

based on normalised slip angles, $\quad \bar{\alpha}_{i}=\frac{g_{i} C_{\alpha 0} \alpha_{i}}{c_{i} p_{i} F_{0}}$

where $i$ refers to front, $f$ or rear $r$ axles, and the slip angles are given by

$$
\alpha_{f}=(-v-a r) / u+\delta, \quad \alpha_{r}=(-v+(L-a) r) / u
$$

Table 1 lists and specifies default values for the model, which is defined in this way in order to normalise the variables which might be identified. In Section $4, \theta$ is chosen as a subset of $\left[a, m, i, p_{f}, g_{f}, c_{f}, e_{f}, p_{r}, g_{r}, c_{r}, e_{r}\right]$ with the remaining parameters set to default values. Since the Kalman filter objective is minimisation of $\operatorname{trace}(\mathbf{P})$, selection of parameters of equal order allows approximately equal priority to be placed on each identified parameter.

\begin{tabular}{|l|c|c|c|}
\hline & param & (default) value & units \\
\hline Wheelbase & $L$ & 2.4 & $\mathrm{~m}$ \\
\hline Normalising Mass & $M_{0}$ & 1050 & $\mathrm{~kg}$ \\
\hline Normalising yaw moment of inertia & $I_{Z Z 0}$ & 1100 & $\mathrm{kgm}$ \\
\hline Normalising peak lateral tyre force (2 tyres) & $F_{0}$ & 4500 & $\mathrm{~N}$ \\
\hline Normalising cornering stiffness (2 tyres) & $C_{\alpha 0}$ & 148.3 & $\mathrm{kN} / \mathrm{rad}$ \\
\hline CG to front axle distance & $a$ & 1 & $\mathrm{~m}$ \\
\hline mass multiplier & $m$ & 1 & - \\
\hline yaw inertia multiplier & $i$ & 1 & - \\
\hline Peak tyre force multiplier & $p_{f,}, p_{r}$ & 1,1 & - \\
\hline $\begin{array}{l}\text { Tyre force gain (cornering stiffness) } \\
\text { multiplier }\end{array}$ & $g_{f}, g_{r}$ & 1,1 & - \\
\hline Tyre model shape parameter (Pacejka, C) & $c_{f}, c_{r}$ & $1.4,1.4$ & - \\
\hline Tyre model shape parameter (Pacejka, E) & $e_{f}, e_{r}$ & $-0.2,-0.2$ & - \\
\hline
\end{tabular}


From equations (26) and (27) the required definition of the IEKF model of equation (14) is

$$
\mathbf{y}=\left[\begin{array}{c}
v \\
r
\end{array}\right], \quad \mathbf{h}_{k}=\left[\begin{array}{c}
v_{k-1}+\left.T\left\{\left(F_{y f}+F_{y r}\right) / m M_{0}-u r\right\}\right|_{k} \\
r_{k-1}+T\left(a F_{y f}-(L-a) F_{y r}\right) /\left.i I_{z Z 0}\right|_{k}
\end{array}\right]
$$

and for implementation, the $\mathbf{H}$ Jacobian is formed using the analytical Math toolbox (Symbolic Maths in Matlab), with the resulting expression pasted into the Kalman filter code.

The source data is generated using a higher order model (see Gordon and Best (2006)), incorporating roll and longitudinal dynamics, and four load dependent friction circle limited tyres with appropriate relaxation lags; the precise model equations and parameter set are provided in the Appendix.

Note that the detail of the model, and even to some extent the accuracy of the source data itself is of secondary importance; the study should reveal similar results for any suitably formulated high order model, or indeed for an actual test vehicle. It is the extent to which the identified parameters can approximate the source model within the context of the simpler identification model which is key here, along with its stability and robustness to measurement error.

\section{RESULTS}

\subsection{Experiment 1 : IEKF performance and (low g) model identification}

Here the aim is to understand the value of the IEKF as an off-line identification tool, and we do this first in the context of identifying the principal parameters governing the linear behaviour,

$$
\boldsymbol{\theta}=\left[\begin{array}{lll}
m & i & a
\end{array}\right]^{T}
$$

Accordingly, the simulated identification test data is taken from a random steer input at fixed speed $\mathrm{u}=25 \mathrm{~m} / \mathrm{s}$. 60 seconds of gaussian white noise signal is filtered in the frequency domain to remove all frequencies above $5 \mathrm{~Hz}$; this restricts the input frequencies to those which a human driver could be expected to achieve in a real vehicle. The signal amplitude is then scaled to give a Root-Mean-Square (RMS) steer angle $\delta_{\text {RMS }}=0.57^{\circ}$, in order to achieve peaks of around $4 \mathrm{~m} / \mathrm{s}^{2}$ in the source model lateral acceleration and hence maintain the excitation within the linear region of the tyres. The constant forward speed is maintained by a simple proportional feedback control on the applied engine torque.

The source data is applied to the IEKF, with no additional measurement noise - the significant differences between source and identified model structure comprise the only error sources at this stage. Five cases are considered, to establish performance relative to $\tau, \lambda$ and $\boldsymbol{\theta}_{\mathbf{0}}$ choices, and to explore an alternative, fixed $\mathbf{Q} \& \mathbf{S}$ approach; the tests are listed in table 2 , and in all cases the error covariance matrices are initialised nominally, as $\mathbf{Q}_{\mathbf{0}}=10^{-4} \mathbf{I}, \mathbf{S}_{\mathbf{0}}=\mathbf{0}$

\begin{tabular}{|c|c|c|c|c|c|c|}
\hline case & $m_{0}$ & $i_{0}$ & $a_{0}$ & $\tau$ & $\lambda$ & fix Q\&S ? \\
\hline 1 & 1 & 1 & 1 & 30 & 0.01 & no \\
\hline
\end{tabular}




\begin{tabular}{|c|c|c|c|c|c|c|}
\hline 2 & 0.5 & 2 & 0.5 & 30 & 0.01 & no \\
\hline 3 & 1 & 1 & 1 & 10 & 0.01 & no \\
\hline 4 & 1 & 1 & 1 & 30 & 0.07 & no \\
\hline 5 & 1 & 1 & 1 & 30 & 0.01 & yes \\
\hline
\end{tabular}

Table 2 : IEKF Parameter and Initialisation Settings

Given the potential for real-time configuration of the IEKF, to establish an averaged result the source data is repeatedly passed through the filter, with the free variables allowed to vary continuously throughout. Figures 1 and 2 show results for all five cases, and 10 iterations are shown to illustrate convergence.

Figure 1 shows the parameter variations, whilst figure 2 illustrates the variations of selected, but typical components within the error matrices. In figure 2 it should be noted that values in $\mathbf{P}$ and $\mathbf{Q}$ reflect the filter's estimate of error, whereas $\mathbf{R}_{\mathbf{k}}$ provides a more verifiable measure of performance - the filter vs source error in the measurements (the innovations). Figure 2(c) shows trace $(\mathbf{R})$ to show the aggregated performance over the two sensors.

[Figures 1 and 2 here]

As a further, independent measure of performance, the final converged parameter sets are fixed and applied in an open-loop validation of the identification model, with $u_{k}$ and $\delta_{k}$ provided as for the source model, over a separate but similar band limited white noise test. Table 3 shows a comparison of validation time histories, for each case along with the nominal parameter setting $\theta_{0}=[1,1,1]^{\mathrm{T}}$, and a typical selection of the related time histories for $\theta_{0}$ and case 1 is also illustrated in Figure 3.

\begin{tabular}{|c|c|c|c|c|c|c|}
\hline $\begin{array}{c}\text { Error variance } \\
\left.(\mathrm{x} \mathrm{10})^{-3}\right)\end{array}$ & $\theta_{0}$ & case 1 & case 2 & case 3 & case 4 & case 5 \\
\hline in $\hat{v}$ & 4.95 & 1.50 & 1.50 & 1.50 & 2.18 & 2.01 \\
\hline in $\hat{r}$ & 0.778 & 0.455 & 0.455 & 0.458 & 0.899 & 0.711 \\
\hline
\end{tabular}

Table 3 : Validation; Identified Model Performance

[Figure 3 here]

Across the results, cases $1-4$ show that the IEKF performs well, and figure 2 shows an expected decrease in $\mathbf{Q}_{\mathbf{k}}$ towards zero, with corresponding decreases to consistent low values in $\mathbf{P}_{\mathbf{k}}$ and $\mathbf{R}_{\mathbf{k}}$. Case 1 achieves the best results, showing a 70\% improvement in $\hat{v}$ and a $42 \%$ improvement in $\hat{r}$, and this is the reference case. It is interesting to note that the improvements come by over-estimation of the mass and inertia; the source model includes tyre lags, which result in higher peak $v$, and a small phase lag in $r$ compared with the identification model. Figure 3 shows that the higher $m$ and $i$ in the identified model cause higher $v$, and also reduce the phase error in $r$, though at some cost in accuracy in yaw rate peaks. Of course, a better identification model could include a relaxation parameter in the tyre model, to more suitably eliminate this problem, but here we are deliberately restricting the study to identification of the best parameters within the restricted model, so the result is correct. 
Cases 1 and 2 show that a consistent result is obtained independently of the initial parameter choice, and this has also been verified for other random choices - note however, that as physically interpreted parameters, very large diversions from the nominal would not be expected - indeed these would indicate poor conditioning in the choice of parameter set. (As an example, if the wider choice $\boldsymbol{\theta}=\left[\begin{array}{llll}m & i & a & g\end{array}\right]^{T}$ is made - with $g$ applied equally to both front and rear - it is clear by inspection of equations (26)-(29) that the identification model becomes rank deficient, and for that case the IEKF responds by returning values of $m, i$ and $g$ which tend towards zero.)

The results are not particularly sensitive to the IEKF tuning parameters $\tau$ and $\lambda$. $\tau$ reflects the 'memory' of the past error matrix estimates, and this can be set according to the context and duration of the test data; here $\tau$ is set to achieve an averaging of the errors across the 60 second test. Note that the rate of performance improvement, seen in figure 2(a) and 2(b) is principally affected by $\tau$. For off-line identification from a fixed data set, it is wise to choose a relatively high $\tau$, to provide a slow, consistent parameter convergence. Optimisation of $\tau$ is not necessary however; very high settings (higher than $30 \mathrm{~s}$ in the cases considered) offer little benefit in ultimate parameter values, serving only to increase the number of data set iterations required.

Case 4 shows the effect of varying $\lambda$, with $\lambda=0.07$ chosen close to the limit of filter stability. High $\lambda$ settings generate a higher expectation of parameter error in the filter, and hence induce larger Kalman gains $\mathbf{K}$, and stronger measurement error feedback. This can make the filter respond more quickly, as we will see later in experiment 3, but the final parameter choices are weaker here, as is confirmed in Figure 2(a) and 2(b). For this time-averaged system identification, lower $\lambda$ settings are ideal, the only disadvantage of very low settings being the need for more data set iteration.

The final case considers $\mathbf{Q}$ and $\mathbf{S}$ fixed at their initial values, the purpose being to explore the self-regulating nature of the IEKF; there is perhaps some cause for concern in that in selfregulating $\mathbf{Q}$ and $\mathbf{S}$, the IEKF determines its expectation of parameter variation error solely on the basis of past parameter variations, caused by the filter itself. Having resolved the obvious stability issue by setting $\lambda<1$, it is true that the filter will inevitably reduce $|\mathbf{Q}|$ by definition. Case 5 shows that the alternative choice of restricting $\mathbf{Q}$ and $\mathbf{S}$ is of no benefit however. Of course, $\mathbf{Q}_{\mathbf{0}}$ and $\mathbf{S}_{\mathbf{0}}$ are suboptimal fixed choices, and better results might be achieved by their tuning, but by fixing these values, the reduction in $\mathbf{R}$ causes the filter to become increasingly unstable, for reasons similar to case 4.

Interestingly, the IEKF does not generate a strictly optimal solution for $\theta$, because the expectation of zero time correlation - equation (4) - will not generally be true. (An example is the phase error seen earlier in $r$ ). It is therefore worth bench-marking the quality of identification, and this can readily be done by comparing against the most commonly used alternative method, Ordinary Least Squares (OLS). From equations (26) and (27) we can write

$$
\begin{gathered}
\dot{v}+u r=\left[\left(F_{y f}+F_{y r}\right) / M_{0}\right] \cdot[1 / m] \\
\dot{r}=\left[\left(a F_{y f}-(L-a) F_{y r}\right) / I_{z Z 0}\right] \cdot[1 / i]
\end{gathered}
$$


which provide linear-in-the-parameter equations of the form $y=[\underline{u}] .[\theta]$ that can be solved by the OLS formula

$$
\hat{\theta}=\left(\underline{u}^{T} \underline{u}\right)^{-1} \underline{u}^{T} \underline{y}
$$

In practice, a recursive least squares (RLS) form could be used, and this would be more directly comparable in operation to IEKF, but the final identified parameters should be identical by OLS or RLS. By repeating the above IEKF experiment using the reduced sensor set, $\boldsymbol{\theta}=[m, i]^{\mathrm{T}}$, and conducting validations as before, OLS is compared with IEKF in table 4.

\begin{tabular}{|c|c|c|}
\hline $\begin{array}{c}\text { Error variance } \\
\left(\mathrm{x} 10^{-3}\right)\end{array}$ & in $\hat{v}$ & in $\hat{r}$ \\
\hline IEKF & 2.27 & 0.605 \\
\hline OLS & 2.32 & 0.548 \\
\hline
\end{tabular}

\begin{tabular}{|c|c|c|}
\hline $\begin{array}{c}\text { Identified } \\
\text { values }\end{array}$ & $m$ & $i$ \\
\hline IEKF & 1.51 & 2.15 \\
\hline OLS & 1.44 & 1.72 \\
\hline
\end{tabular}

Table 4 : Validation of IEKF against Ordinary Least Squares

Note that the IEKF does not perform better than OLS in this experiment - rather the results are very similar. (A measure equivalent to trace $(\mathbf{R})$ is given by the sum of the $\hat{v}$ and $\hat{r}$ error variances.) The suboptimal influence of time correlated errors in IEKF appears to have a similar effect to the bias errors caused by error in the regression sequence, $u(t)$, in OLS. And this is reasonable, given that both methods aim to minimise the square of the error sequence. However IEKF provides a significant advantage over OLS in that the equations do not have to be posed in a linear-in-the-parameters form; OLS can not be used to estimate the $\theta=[m, i, a]^{\mathrm{T}}$ set used earlier, nor could it estimate tyre parameters for the Pacejka model.

\subsection{Experiment 2 : Tyre model (high g) identification and sensor noise}

To explore the full nonlinear range of vehicle handling, a more extreme test is now considered. The same white noise process is employed, now scaled up to $\delta_{\text {RMS }}=2.3^{\circ}$ to induce peak lateral accelerations of $8 \mathrm{~m} / \mathrm{s} 2$. The parameter set is now

$$
\boldsymbol{\theta}=\left[\begin{array}{llllllll}
p_{f} & g_{f} & c_{f} & e_{f} & p_{r} & g_{r} & c_{r} & e_{r}
\end{array}\right]^{T}
$$

So a smoothly nonlinear tyre model is identified, separately for front and rear axles. Note that the inertia and CG parameters are now excluded, to avoid an underdetermined system.

The effect of more extreme errors is also considered here, by the addition of a random noise sequence to the sensor measurements; this is white noise, filtered in the frequency domain, to remove all frequencies above $10 \mathrm{~Hz}$. The filtering is necessary because the sensor equations (31) consider variations over each filter timestep, so high frequency noise emulates an effect of very high tyre forces which fatally corrupts the estimate. However, with a view to practical implementation of IEKF, it is entirely reasonable to filter the whole signal prior to identification, since the dynamics of interest lie exclusively in the $0-5 \mathrm{~Hz}$ range. The noise is added proportionally to both $v$ and $r$ measurements, and the extreme case of a $50 \%$ noise / signal ratio is applied.

The IEKF used the setting of case 1 in experiment 1, and figure 4 illustrates the parameter convergence over a very large number of iterations; 500 iterations took 125 minutes of processing in Matlab, on the mid-range PC used here. However, the $\mathbf{R}$ matrix is static 
throughout most of the iterations, with its trace reducing from $1.153 \times 10^{-4}$ to $0.993 \times 10^{-4}$ after the $10^{\text {th }}$ iteration, and then ultimately to $0.990 \times 10^{-4}$ by the $500^{\text {th }}$. In combination, the parameter changes make very little difference to the identified tyre curve. Figure 5 shows this by illustrating the resulting tyre force model at the same three iterations in the convergence; in practice it is necessary only to iterate on the dataset 10 to 15 times (which would take $2-3$ mins of processing).

Finally, table 5 summarises the validation results for open-loop model simulations. As well as validating the IEKF process for tyre model identification, these results also show excellent robustness to the high sensor noise case.

\begin{tabular}{|c|c|c|c|c|}
\hline & \multicolumn{2}{|c|}{ Noise free } & \multicolumn{2}{c|}{$50 \%$ Noise case } \\
\hline $\begin{array}{c}\text { Error variance } \\
\left(\mathrm{x} \mathrm{10} 0^{-3}\right)\end{array}$ & $\theta_{0}$ & IEKF & $\theta_{0}$ & IEKF \\
\hline in $\hat{v}$ & 1.800 & 0.995 & 2.483 & 1.287 \\
\hline in $\hat{r}$ & 0.164 & 0.091 & 0.331 & 0.245 \\
\hline
\end{tabular}

Table 5 : Validation of IEKF tyre model with and without noise

[Figures 4 and 5 here]

\subsection{Experiment 3 : Real-time parameter identification and the combined Kalman filter}

Providing due consideration is given to selection of $\lambda$, it is possible to operate the IEKF in an alternative, fast mode. To examine this, and also test a further error condition, a series of manoeuvres are conducted to provide both lateral and longitudinal excitation to the source model. The test comprises two step steer events followed by combined steering with acceleration, and finally hard braking. This is repeated, first with low magnitude inputs, then with higher magnitudes, and in between these a lane-change manoeuvre is also applied.

The IEKF is set with a nominal, short error covariance 'memory', $\tau=1$, and with $\lambda=0.05$ to induce fast adaptation, and the results can be seen in figure 6 . There is a significant and rapid change in parameters at the onset of the step steer manoeuvres. Note how the cornering stiffness parameter, $g$ adapts for the low magnitude step at 5 seconds, whereas the peak force and shape parameters $(p$ and $c$ ) are altered for the higher magnitude step at 37 seconds. There is also an expected reduction in peak force between 20 and 35 seconds, where the longitudinal force demand from the tyres reduces the lateral force capability.

As a final illustration of the further scope of the IEKF, figure 6 also shows the result of coupling the identifying filter to a traditional EKF state estimator. The IEKF operates entirely independently, and at each time step provides a revised parameter set to the EKF. This runs according to equations (6) - (14), using a fixed design set of noise matrices that were determined by simulation of the error sequence, generated by comparing source model results with open-loop simulations of the identification model, operating using the nominal parameter set of table 1 , on the white noise, constant speed test used in experiment 1 .

[Figure 6 here] 
The result is a filter with relatively low Kalman gains, and hence the performance improves noticeably with the improving parameter selection as we would expect. Although we could derive a more accurate combined filter, or a more realistic test by exploring sensor noise and design matrix choices, this simple experiment nicely illustrates the value of the model within the Kalman filter, and the scope of the IEKF as a coupled real-time tool as well as an off-line tool for identification.

\section{CONCLUDING REMARKS}

The results show the IEKF to be a versatile, stable and easily configured process for both fixed and time-varying estimation of model parameters. The technique has been shown to be robust to high noise/signal ratios where the noise is uncorrelated but focussed on the frequency range of interest. In conclusion, IEKF should be well suited to rapid prototyping of simplified models for test vehicles and for on-line use within an integrated chassis control structure. Also, as this filter is independent of any specific observer or controller, it is well suited to providing real-time vehicle parameter variations to several independent and/or coordinating controllers.

The only significant disadvantage is the need for a sensor set which spans the state space of the identification model, and this restricts its current use to relatively expensive GPS / inertia based acquisition systems. However, with future technology cost reductions and the certain future integration of GPS within vehicles, this hurdle should be overcome within the medium term.

The next steps for development are to conduct vehicle-based experiments, and to explore slightly higher order identification model structures, particularly with a view to providing ride parameters, and combined lateral / longitudinal tyre force models. Success in achieving these two goals will establish IEKF as a valuable tool for future integrated chassis control development.

\section{REFERENCES}

Arndt, C., Karidas, J., Busch, R. (2005) 'Estimating Non-measured Vehicle States with an Extended Linearised Kalman Filter', Review of Automotive Engineering, vol 26, no. 1, pp. 91 - 98.

Best M.C., Gordon T.J. and Dixon P.J. (2000) 'An Extended Adaptive Kalman Filter for Real-time State Estimation of Vehicle Handling Dynamics' Vehicle System Dynamics, vol 34, no 1, pp 5775.

Best M.C. and Gordon T.J. (1994) 'A Factorial Analysis of Kalman Filtering for Semi-Active Vehicle Suspension Control' proceedings from the ESDA / ASME European Joint Conference on Engineering Systems Design and Analysis, London, U.K., July 1994

Gelb, A. (Ed.) (1974) Applied Optimal Estimation. MIT Press, Cambridge Mass..

Gordon, T.J., and Best, M.C. (2006) 'On the Synthesis of Driver Inputs for the Simulation of Closedloop Handling Manoeuvres', International Journal of Vehicle Design, vol 40, no 1/2/3, pp 5276. 
Hancock, M.J., Williams, R.A., Gordon, T.J., and Best, M.C. (2005) 'Comparison of Braking and Differential Control of Interactive Yaw-Sideslip Dynamics', Proceedings of the Institution of Mechanical Engineers, Part D, March 2005, vol.219, no.D3, pp. 309-27.

Haykin, S. (Ed.) (2001) Kalman filtering and Neural Networks, New York: John Wiley.

Qu, Q., Zu, J.W. (2005) 'Variable structure model following control of four-wheel steering vehicle', International Journal of Vehicle Design, vol 37, no 4, pp 291 - 310.

Wenzel, T.A., Burnham, K.J., Blundell, M.V. and Williams, R.A. (2006) 'Dual Extended Kalman Filter for Vehicle State and Parameter Estimation', Vehicle System Dynamics vol 44(2), pp. 153171

\section{APPENDIX}

\section{Source model dynamics}

The source model is based on the well known three degree of freedom model, simulating yaw, roll, and sideslip using a load dependent, combined-slip Pacejka tyre model. A fourth, longitudinal degree of freedom is also included, as are additional dynamics for wheel-spin, and first-order lags for tyre relaxation.

The principal equations of motion are

longitudinal:

$$
M \dot{U}=\sum_{i=1,4} F_{x i}+M r V+M h r p
$$

lateral:

$$
M \dot{V}+M h \dot{p}=\sum_{i=1,4} F_{y i}-M U r
$$

yaw:

$$
I_{z z} \dot{r}=a \sum_{i=1,2} F_{y i}-b \sum_{i=3,4} F_{y i}
$$

roll:

$$
-I_{x z} \dot{r}+M h \dot{V}+I_{x x} \dot{p}=-M h U r-\left(B_{f}+B_{r}\right) p+\left(M g h-K_{f}-K_{r}\right) \phi
$$

roll kinematics:

$$
\dot{\phi}=p
$$

Standard SAE axes are used (Gillespie 1992) fixed relative to the vehicle wheelbase; the wheels are labelled (1-4) in ascending order as (front-left, front-right, rear-left, rear-right). The principal notation and parameter values are given in Table A1.

The forces $\left(F_{x i}, F_{y i}\right)$ controlling the vehicle motion allow for large steer angles

$$
\begin{array}{ll}
F_{x 1,2}=F_{t x 1,2}^{*} \cos \delta-F_{t y 1,2}^{*} \sin \delta, & F_{x 3,4}=F_{t x 3,4}^{*} \\
F_{y 1,2}=F_{t y 1,2}^{*} \cos \delta-F_{t x 1,2}^{*} \sin \delta, & F_{y 3,4}=F_{t y 3,4}^{*}
\end{array}
$$

based on lagged tyre forces, where each of the 8 elements are lagged to simulate relaxation within the tyre

$$
\dot{F}_{t x / y, i}^{*}=\tau^{-1}\left(F_{t x / y, i}-F_{t x / y, i}^{*}\right)
$$


where the tyre forces $\left(F_{t x i}, F_{t y i}\right)$ are modelled according to the Pacejka magic formula

$$
P(\alpha)=P(\alpha ; B, C, D, E) \equiv D \sin \left(C \tan ^{-1}\left(B \alpha-E\left(B \alpha-\tan ^{-1} B \alpha\right)\right)\right)
$$

using normalized slip and isotropic similarity scaling (Milliken and Milliken 1995, Pacejka 2002). In more detail, the normalized slip vector is

$$
\mathbf{k}=\left(\begin{array}{l}
k_{x} \\
k_{y}
\end{array}\right)=\frac{C_{\alpha}}{F_{p}}\left(\begin{array}{c}
s \\
\tan \alpha
\end{array}\right)
$$

where $s$ is the longitudinal slip ratio, and $\alpha$ is the slip angle. The friction circle at each tyre contact patch is defined by the following simple analytic function of vertical load $w$

$$
\sqrt{F_{t x}^{2}+F_{t y}^{2}} \leq F_{p}(w)=\frac{w}{1+(3 w / 2 M g)^{3}}
$$

and the load-dependent cornering/longitudinal stiffness for each tyre is of the form

$$
C_{\alpha}(w)=c_{1}\left(1-e^{-w / c_{2}}\right)
$$

(see Table 1 for values) and the resulting tyre force vector is

$$
\left(\begin{array}{l}
F_{t x} \\
F_{t y}
\end{array}\right)=P(|\mathbf{k}|) \frac{F_{p}}{|\mathbf{k}|}\left(\begin{array}{l}
k_{x} \\
k_{y}
\end{array}\right)
$$

Vertical tyre loads are calculated from static weight distribution, and modified to accommodate lateral load transfer according to :

$$
\Delta_{l a t} w_{f / r}=\frac{\sum F_{y f / r} h_{R}+K_{f / r} \phi+B_{f / r} p+M g h \sin \phi}{t_{f / r}}
$$

And to accommodate longitudinal load transfer, approximated as :

$$
\Delta_{\text {long }} w=\frac{\sum_{i} F_{x i} \cdot\left(h_{R}+h\right)}{(a+b)}
$$

This tyre model is only broadly representative of real tyre behaviour, but is thought to incorporate sufficiently realistic aspects of force saturation and load dependence to properly test the IEKF identification process. Wheel rotational dynamics are modelled as

$$
\dot{\omega}_{i}=I_{w}^{-1}\left(T_{i}-r_{r} F_{t x i}\right)
$$

where $T_{i}$ is the drive torque (positive) or brake torque (negative) torque, and $I_{w}=5 \mathrm{~kg} \mathrm{~m}^{2}$ is the nominal wheel inertia, incorporating tyre, engine and driveline components. The drive 
torques $T_{i}$ are directly commanded and apportioned equally between left and right wheels, and in the case of brake torque, apportioned in the ratio 60:40 between front and rear axles. Drive torque is apportioned entirely to the front (FWD). 


\begin{tabular}{|ll|}
\hline \multicolumn{2}{|c|}{ States and Dynamic Variables $($ units $)$} \\
\hline$U$ & vehicle forward velocity $\left(\mathrm{m} \mathrm{s}^{-1}\right)$ \\
$V$ & sideslip velocity $\left(\mathrm{m} \mathrm{s}^{-1}\right)$ \\
$p$ & roll angular velocity $\left(\mathrm{rad} \mathrm{s}^{-1}\right)$ \\
$r$ & yaw angular velocity $\left(\mathrm{rad} \mathrm{s}^{-1}\right)$ \\
$\phi$ & roll angle $(\mathrm{rad})$ \\
$\theta$ & yaw angle $(\mathrm{rad})$ \\
$T_{i}$ & driveline or brake torque at wheel $i$ \\
$\omega$ & wheel angular velocity $\left(\mathrm{rad} \mathrm{s}^{-1}\right)$ \\
$w$ & magnitude of tyre vertical load $(\mathrm{N})$ \\
\hline Parameters & (value, units) \\
\hline$I_{x x}$ & body roll moment of inertia $\left(175 \mathrm{kgm}^{2}\right)$ \\
$I_{z z}$ & yaw moment of inertia $\left(1100 \mathrm{kgm}^{2}\right)$ \\
$I_{x z}$ & roll/yaw product of inertia $\left(0 \mathrm{kgm}^{2}\right)$ \\
$I_{w}$ & wheel (plus associated driveline $)$ moment of inertia $\left(5 \mathrm{kgm}^{2}\right)$ \\
$M$ & vehicle mass $(1045 \mathrm{~kg})$ \\
$a$ & longitudinal Distance of C of G to front axle $(1.0 \mathrm{~m})$ \\
$b$ & longitudinal Distance of C of G to rear axle $(1.4 \mathrm{~m})$ \\
$h$ & C of G height above roll axis $(0.42 \mathrm{~m})$ \\
$h_{f}$ & suspension roll centre height above ground $(0.19 \mathrm{~m})$ \\
$t_{f}$ & front track $(1.35 \mathrm{~m})$ \\
$t_{r}$ & rear track $(1.35 \mathrm{~m})$ \\
$r_{r}$ & tyre rolling radius $(0.3 \mathrm{~m})$ \\
$K_{f}$ & front roll stiffness $(19.7 \mathrm{kNm} / \mathrm{rad})$ \\
$K_{r}$ & rear roll stiffness $(9.7 \mathrm{kNm} / \mathrm{rad})$ \\
$B_{f}$ & front roll damping $(1000 \mathrm{Nms} / \mathrm{rad})$ \\
$B_{r}$ & rear roll damping $(911 \mathrm{Nms} / \mathrm{rad})$ \\
$B, C, D, E$ & Pacejka formula coefficients $(1.0,1.4,1.0,-0.2-\mathrm{dimensionless})$ \\
$c_{1}, c_{2}$ & cornering stiffness parameters $(69 \mathrm{kN} / \mathrm{rad}, 1.4 \mathrm{kN})$ \\
$\tau$ & time-constant for tyre relaxation $(0.025 \mathrm{sec})$ \\
\hline
\end{tabular}

Table A1. Source Vehicle Model Notation and Parameter Values 


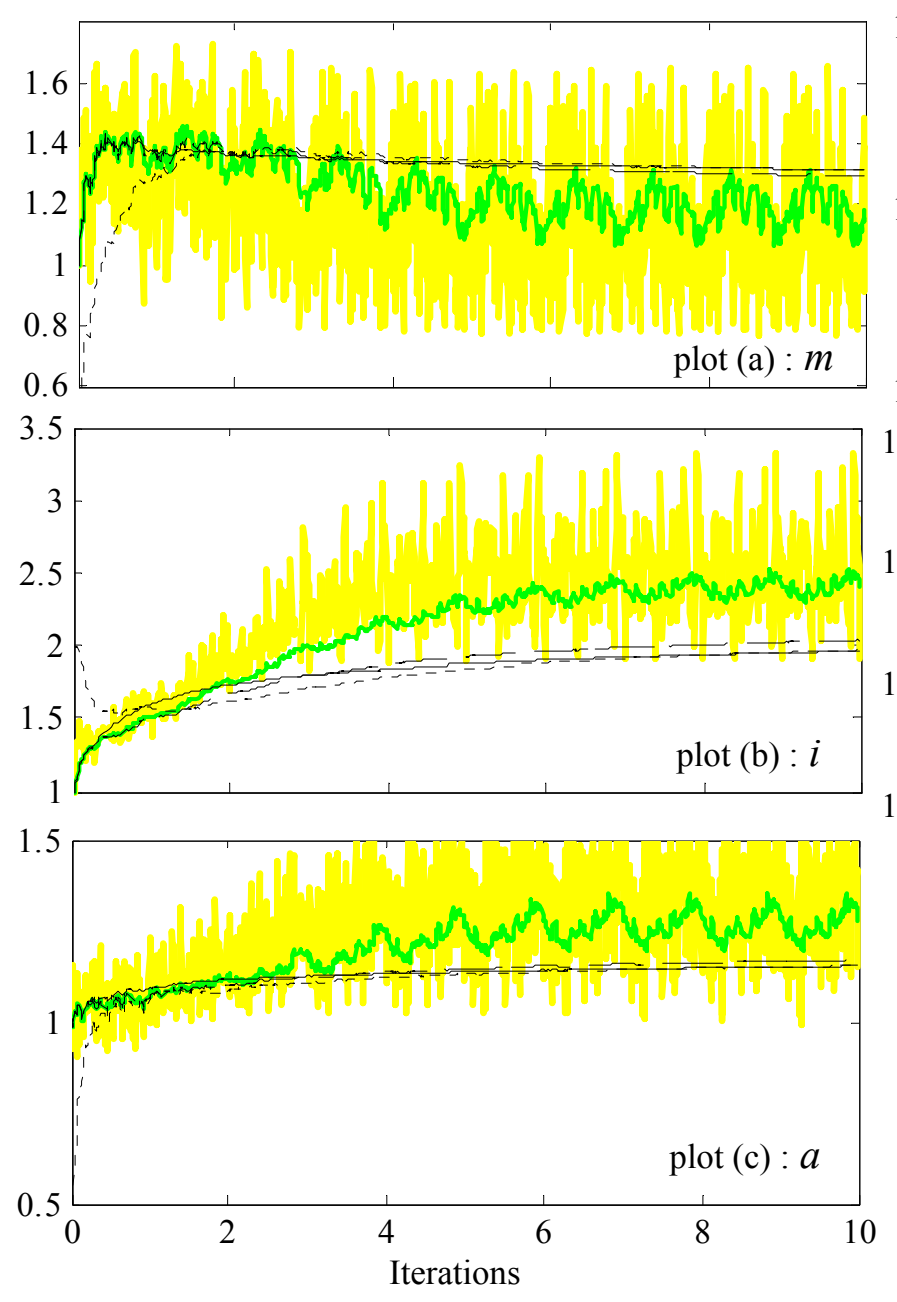

Figure 1 : Parameter convergence convergence
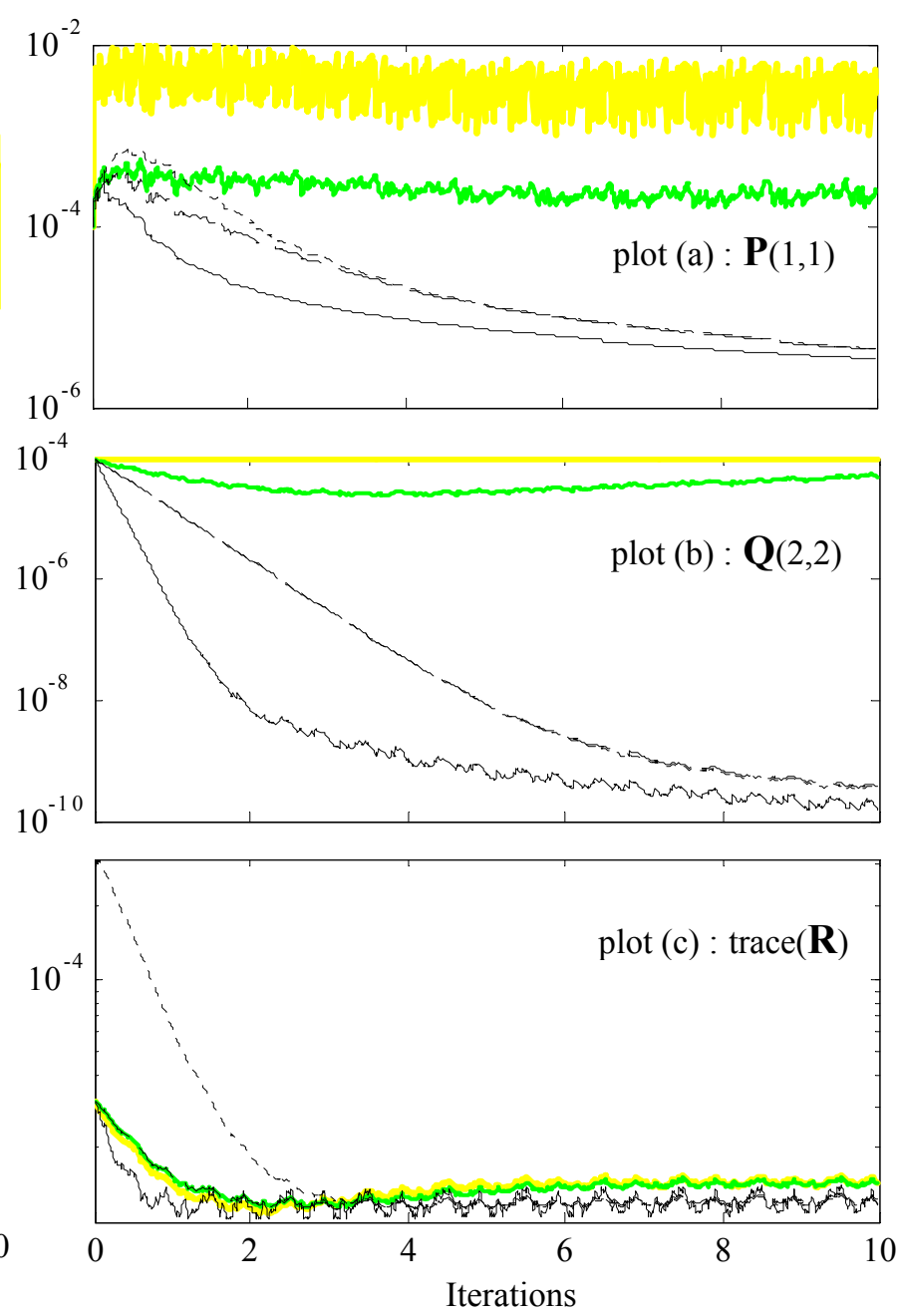

Figure 2 : Error covariance 


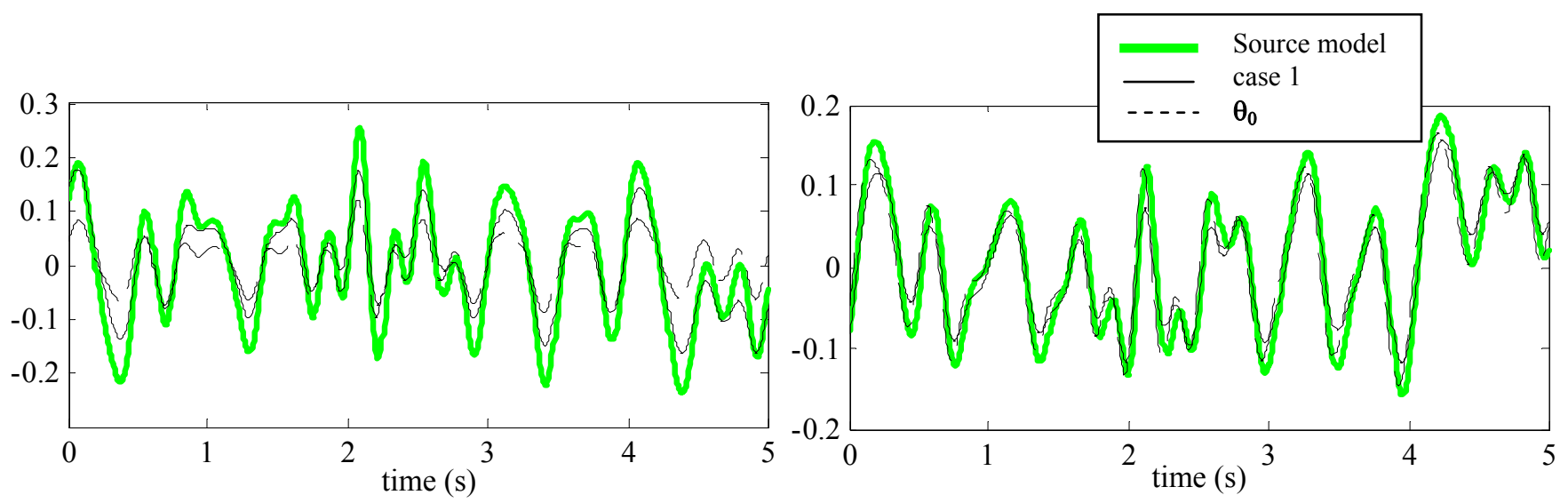

Figure 3 : Model Performance in lateral velocity $(\mathrm{m} / \mathrm{s}$, left) and yaw rate ( $\mathrm{rad} / \mathrm{s}$, right) 

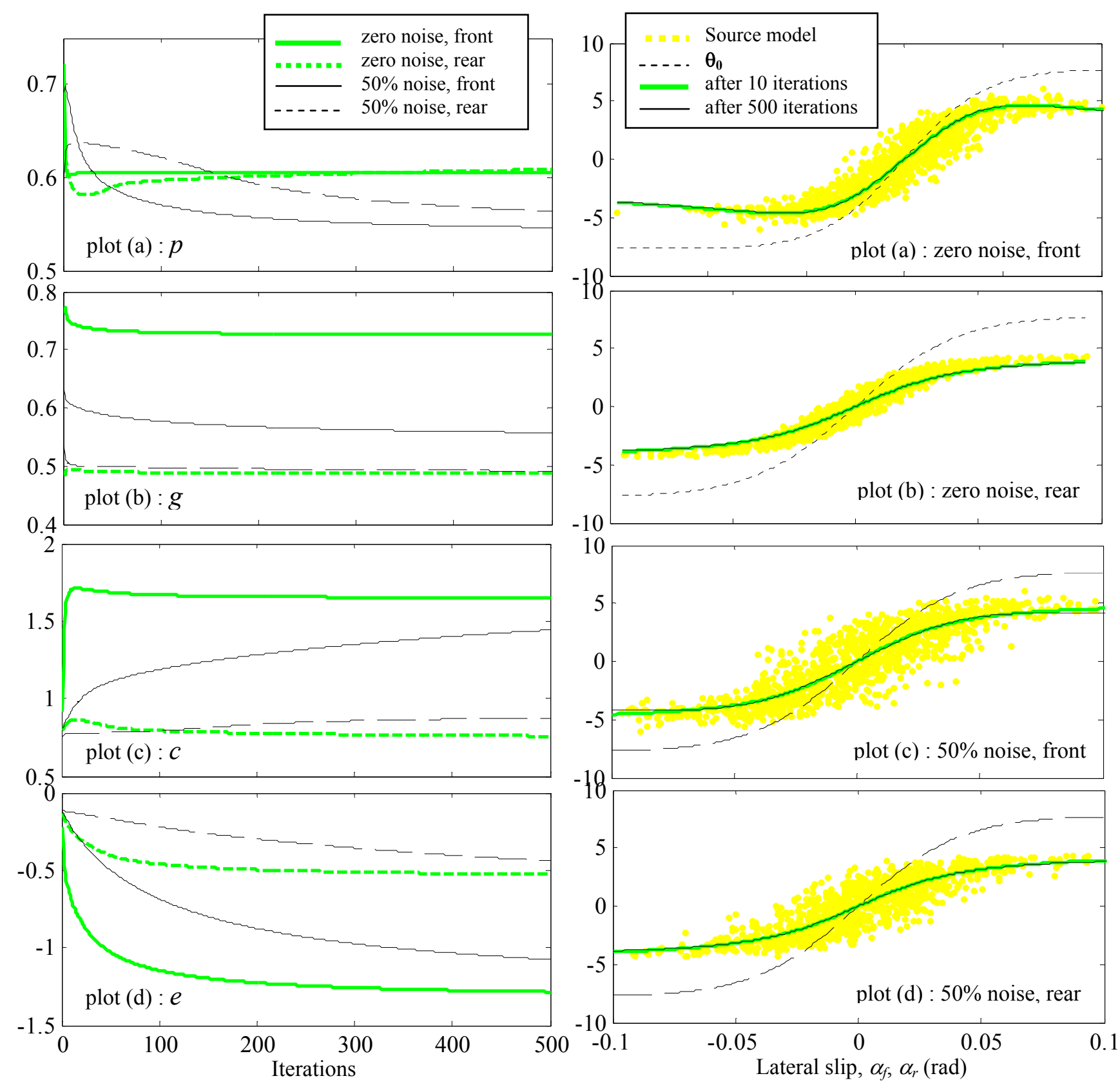

Figure 4 : Tyre parameter convergence

Figure 5 : Tyre forces $(k N)$ model fit 


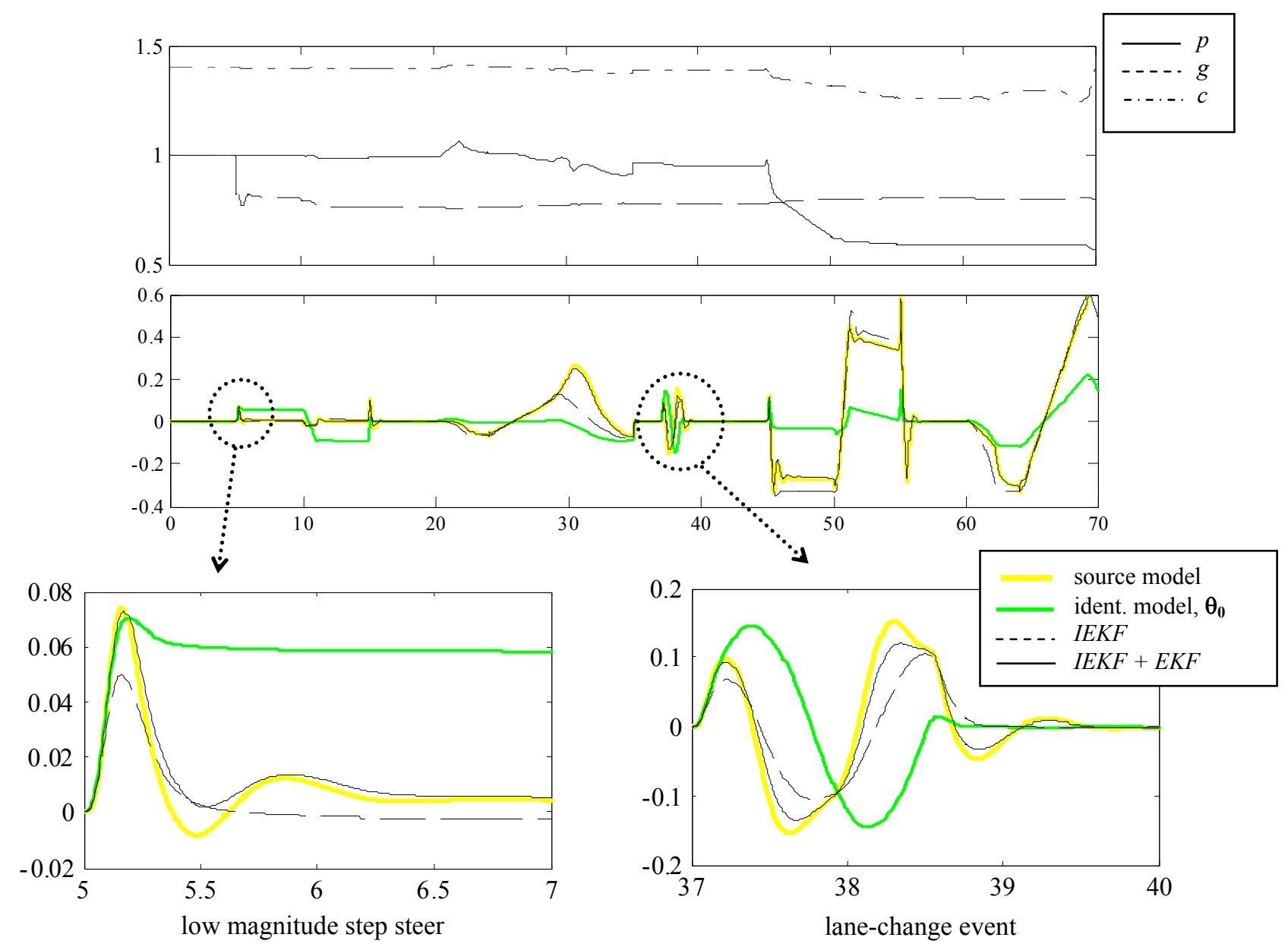

Figure 6 : Real-time IEKF application, alone and in conjunction with EKF state estimator 